\title{
Armazenamento de ameixas ‘Laetitia’ em atmosfera modificada
}

\author{
Storage of 'Laetitia' plums under modified atmosphere
}

\author{
Cristiano André Steffens ${ }^{\mathrm{I}^{*}}$ Cassandro Vidal Talamini do Amarante ${ }^{\mathrm{I}}$ \\ Erlani de Oliveira Alves ${ }^{\text {I }}$ Hélio Tanaka' Auri Brackmann ${ }^{\text {II }}$ Vanderlei Both ${ }^{\text {II }}$
}

RESUMO

Objetivou-se avaliar o efeito da atmosfera modificada (AM) na manutenção da qualidade físico-química de ameixas da cultivar 'Laetitia'. Os tratamentos avaliados foram: armazenamento refrigerado $\left(A R ; 21,0 \mathrm{kPaO} \mathrm{O}_{2}+0,03 \mathrm{kPa}\right.$

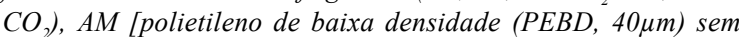
perfuração], $\mathrm{AM}$ com adição de $10 \mathrm{kPa}$ de $\mathrm{CO}_{2}$ e $\mathrm{AM} \mathrm{com}$ quatro perfurações de $2,0 \mathrm{~mm}$ de diâmetro. Os tratamentos AM, AM com perfurações e AM com adição de $\mathrm{CO}_{2}$ proporcionaram as pressões parciais médias de $1,9 \mathrm{kPa} \mathrm{O}_{2}+$ $15,2 \mathrm{kPa} \mathrm{CO}_{2}, 8,1 \mathrm{kPaO},+9,6 \mathrm{kPa} \mathrm{CO}_{2}$ e 4,1 $\mathrm{kPaO},+16,0 \mathrm{kPa}$ $\mathrm{CO}_{2}$, respectivamente. Ameixas armazenadas em $A R$ apresentaram menores valores de firmeza de polpa e força para ruptura da casca, bem como coloração da epiderme mais vermelha e maior incidencia de rachaduras na saída da câmara. A força para penetração da polpa e a resistência do fruto à compressão, na saída da câmara, foram maiores nos frutos armazenados em AM com perfuração, não havendo diferenças entre as condições de AM após quatro dias em condição ambiente. A acidez titulável foi mais elevada em todas as condições de AM, na saída da câmara. A incidência de podridões foi menor nos frutos armazenados em AM com perfurações. A taxa respiratória foi mais elevada nos frutos armazenados em AM na saída da câmara, mas não após quatro dias em condição ambiente. A incidência de degenerescência da polpa foi elevada em todos os tratamentos, porém menor em AR e AM com perfurações.

Palavras-chave: Prunus salicina, pós-colheita, degenerescência da polpa, qualidade.

\section{ABSTRACT}

This research was carried out to assess the effects of modified atmosphere packing (MAP) conditions on physicochemical quality preservation of $\mathrm{cv}$. 'Laetitia plums'.
Fruit were cold stored (CS; 21.0kPa $\mathrm{O}_{2}+0.03 \mathrm{kPa} \mathrm{CO}_{2}$ ), MAP

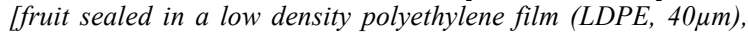
without perforation], MAP with the addition of $\mathrm{CO}_{2}(10 \mathrm{kPa})$ inside the package, and MAP with perforated film (diameter of perforations $=2.0 \mathrm{~mm})$. During cold storage, the treatments MAP, MAP with perforated LDPE film, and MAP with the addition of $\mathrm{CO}_{2}$ had mean partial pressures of $1.9 \mathrm{kPaO} \mathrm{O}_{2}+$ $15.2 \mathrm{kPa} \mathrm{CO}_{2}, 8.1 \mathrm{kPaO}+9.6 \mathrm{kPa} \mathrm{CO}_{2}$ e $4.1 \mathrm{kPaO}+16.0 \mathrm{kPa}$ $\mathrm{CO}_{2}$, respectively. Plums stored under CS had the lowest values of flesh firmness and force for skin penetration (measured with a texturometer), as well as less developed skin red color and higher incidence of skin cracking when removed from cold storage. At removal from cold storage, the force for flesh penetration and for fruit compression (measured with a texturometer) was higher for MAP with perforated LDPE film. However, after four days under ambient conditions, there was no significant difference between MAP treatments for these attributes. Titratable acidity assessed at removal from cold storage was higher in fruit left under MA than CS. The incidence of decays was lower in fruit left under MAP with perforated LDPE film. Respiration was higher in fruit under MAP than CS when assessed at removal from cold storage, but not after four days at ambient temperature. 'Laetitia' plums exhibit a high incidence of flesh browning, and this disorder was reduced in fruit under CS and MAP with perforated LDPE film.

Key words: Prunus salicina, postharvest, flesh browning, quality.

\section{INTRODUÇÃO}

Atualmente, o armazenamento de ameixas é realizado sob refrigeração, sistema de armazenamento que permite a conservação dos frutos por no máximo 30 dias (BRACKMANN et al., 2001). No entanto, muitos

IDepartamento de Agronomia, Universidade do Estado de Santa Catarina (UDESC). Av. Luis de Camões, 2090, Bairro Conta Dinheiro, 88520-000, Lajes, Sc, Brasil. E-mail: steffens@cav.udesc.br. *Autor para correspondência.

IIDepartamento de Fitotecnia, Universidade Federal de Santa Maria (UFSM), Santa Maria, RS, Brasil. 
frutos apresentam a sua qualidade comprometida após o armazenamento, ocorrendo grandes perdas. Além disso, frutos de baixa qualidade após o armazenamento poderão apresentar problemas de comercialização, devido à entrada de ameixas oriundas do Chile, com melhor qualidade. Sendo assim, é de extrema importância que os frutos, após o seu armazenamento, mantenham uma ótima qualidade para atrair o interesse do consumidor.

O armazenamento em atmosfera modificada (AM), por ser um sistema de armazenamento que causa maior redução no metabolismo celular do que o uso isolado da refrigeração (STEFFENS et al., 2007a), tem possibilitado que alguns frutos sejam armazenados por mais tempo e com melhor manutenção da qualidade (ALI et al., 2004). A AM é uma alternativa que visa incrementar o efeito do frio no armazenamento de frutos, sendo uma técnica bastante prática e menos onerosa que a atmosfera controlada (AC). A redução da temperatura, a diminuição da pressão parcial de $\mathrm{O}_{2} \mathrm{e}$ o aumento da pressão parcial de $\mathrm{CO}_{2}$ por meio da $\mathrm{AM}$, são os principais fatores que contribuem para a manutenção da qualidade do produto e, consequentemente, para a redução de perdas póscolheita (STEFFENS et al., 2007a). Sendo assim, a AM poderia aumentar o período de oferta da ameixa 'Laetitia', bem como possibilitar a comercialização de frutos com melhor qualidade, durante a entressafra.

Contudo, apesar da AM contribuir na manutenção da qualidade de frutos, pode, em alguns casos, induzir o desenvolvimento de distúrbios fisiológicos e a fermentação dos frutos, devido à intensa redução nos níveis de $\mathrm{O}_{2}$ e ao incremento do $\mathrm{CO}_{2}$ (STEFFENS et al., 2006 e 2007b). Em frutos que são altamente perecíveis, como no caso da ameixa 'Laetitia' (ARGENTA et al., 2003), pode-se fazer uso da adição de $\mathrm{CO}_{2}$ no interior da embalagem. A alta pressão parcial de $\mathrm{CO}_{2}^{2}$, no início do armazenamento, faz com que os processos metabólicos, responsáveis pelo amadurecimento do fruto, sejam reduzidos mais rapidamente, além de poder reduzir a ocorrência de podridões (BRACKMANN et al., 2005).

Como atualmente inexistem trabalhos realizados com a ameixa 'Laetitia', demonstrando o seu potencial de armazenamento em AM, este trabalho teve como objetivo avaliar o efeito da AM na manutenção das qualidades físico-químicas dessa cultivar de ameixa.

\section{MATERIAL E MÉTODOS}

As ameixas 'Laetitia' foram colhidas em pomar comercial, de nove anos de idade, com qualidade sanitária excelente, conduzido no sistema "Y", localizado no Município de Lages, Santa Catarina (SC). Após a colheita, os frutos foram transportados para o Laboratório do Núcleo de Pesquisa em Pós-Colheita (NPP) da Universidade Federal de Santa Maria, localizado no Município de Santa Maria, Rio Grande do Sul (RS). No laboratório, os frutos foram selecionados, sendo eliminados aqueles com lesões, defeitos, ferimentos ou dano mecânico e, posteriormente, foi realizada a homogeneização das unidades experimentais.

O delineamento experimental adotado foi o inteiramente casualizado, sendo utilizadas cinco repetições por tratamento e unidade experimental composta por 30 frutos. Os tratamentos utilizados foram armazenamento refrigerado (AR; $21,0 \mathrm{kPa} \mathrm{O}+$ $0,03 \mathrm{kPa} \mathrm{CO}_{2}$ ), atmosfera modificada [AM; polietileno de baixa densidade (PEBD) sem perfuração], AM com adição de $10 \mathrm{kPa}$ de $\mathrm{CO}_{2}$ e AM com quatro perfurações de 2,0mm de diâmetro, na região mediana da embalagem. A embalagem de polietileno de baixa densidade utilizada em todos os tratamentos possuía uma espessura de $40 \mu \mathrm{m}$. Os frutos de todos os tratamentos foram armazenados durante 60 dias em câmara fria $\left(0,5 \pm 0,1^{\circ} \mathrm{C}\right.$ e $95 \pm 2 \%$ de UR).

Durante o armazenamento, a pressão parcial $\mathrm{de}_{2}$ e $\mathrm{CO}_{2}$ no interior das embalagens foi monitorada semanalmente por meio de um analisador de gases marca Agridatalog.

Após 60 dias de armazenamento, os frutos foram transportados para o Laboratório de Pesquisa em Fisiologia e Tecnologia de Pós-Colheita, da Universidade do Estado de Santa Catarina (UDESC), em Lages, SC. As amostras foram divididas em duas subamostras de 15 frutos, uma para análise na saída da câmara e outra para análise após quatro dias de exposição dos frutos em condição ambiente $\left(20 \pm 2^{\circ} \mathrm{C} /\right.$ $60 \pm 5 \%$ de UR). As variáveis analisadas foram: índice de cor vermelha (ICV) e cor $\left(h^{\circ}\right)$ da epiderme, firmeza de polpa, atributos de textura (força para ruptura da casca, penetração da polpa e resistência do fruto à compressão), acidez titulável (AT), sólidos solúveis (SS), taxa respiratória e ocorrência de podridões, rachaduras e degenerescência da polpa. O ICV, os SS, a AT, os atributos de textura e a $h^{\circ}$ foram avaliados segundo metodologia descrita em STEFFENS et al. (2009). A firmeza de polpa, taxa respiratória e ocorrência de podridões, rachaduras e de degenerescência de polpa foram determinadas conforme descrito em STEFFENS et al. (2008).

Antes do armazenamento dos frutos, duas amostras de 15 frutos foram analisadas para determinar as características da qualidade inicial das ameixas, as 
quais apresentaram firmeza de polpa de $42,0 \mathrm{~N}, \mathrm{SS}$ de $9,3^{\circ}$ Brix e AT de $31,2 \mathrm{mEq}$ de ácido cítrico $100 \mathrm{~mL}^{-1}$.

Os dados foram submetidos à análise de variância e ao teste F. Dados em porcentagem foram transformados pela fórmula arco-seno $[(\mathrm{x}+0,5) / 100]^{1 / 2}$ antes de serem submetidos à análise de variância. Para a comparação das médias, adotou-se o teste de Tukey. Em todas as análises estatísticas, foi utilizada probabilidade de erro de $5 \%$.

\section{RESULTADOS E DISCUSSÃO}

Após 60 dias de armazenamento a $0,5^{\circ} \mathrm{C}$, os tratamentos AM, AM com perfurações e AM com adição de $\mathrm{CO}_{2}$ proporcionaram as pressões parciais médias de $\mathrm{O}_{2}+\mathrm{CO}_{2}(\mathrm{kPa})$ de $1,9+15,2,8,1+9,6$ e 4,1 + 16,0 , respectivamente.

A firmeza de polpa, tanto na saída da câmara, como após quatro dias em temperatura ambiente, foi mais elevada nos frutos armazenados em todos os tratamentos com AM. Dentre os tratamentos em AM, a AM com perfurações proporcionou a maior firmeza de polpa (Tabela 1). Trabalhos realizados com pêssego, nectarina (AKBUDAK \& ERIS, 2004), caqui (CIA et al., 2006), manga (JERONIMO et al., 2007) e carambola (ALI et al., 2004) demonstram que o armazenamento em AM mantém maior firmeza de polpa que o armazenamento refrigerado. A firmeza de polpa é considerada um dos atributos de maior importância na qualidade de frutos, já que afeta a resistência ao transporte e ao ataque de microrganismos (JAYAS \& JEYAMKONDAN, 2002; JERONIMO et al., 2007). Em geral, as alterações na consistência dos frutos durante o amadurecimento resultam, predominantemente, da desestruturação da parede celular, que envolve a interação complexa de várias enzimas hidrolíticas (ALI et al., 2004).

A força para ruptura da epiderme foi mais elevada nos frutos de todos os tratamentos em AM (Tabela 1). No entanto, após quatro dias de exposição dos frutos em condição ambiente, os maiores valores foram obtidos nos tratamentos AM com perfurações e AM com adição de $\mathrm{CO}_{2}$. Já a força para penetração da polpa e a resistência do fruto à compressão, avaliadas na saída da câmara, foram maiores nos frutos armazenados em AM com perfurações. Contudo, após quatro dias de exposição dos frutos em condição ambiente, todos os tratamentos em AM apresentaram valores mais elevados desses atributos de textura, não diferindo entre si (Tabela 1). A manutenção da textura nos frutos armazenados em AM deve-se provavelmente ao retardo no amadurecimento dos frutos e à atividade de enzimas hidrolíticas de parede celular, devido à inibição da respiração e da síntese e da ação do etileno, pelo baixo $\mathrm{O}_{2}$ e pelo alto $\mathrm{CO}_{2}$ (JAYAS \& JEYAMKONDAN ${ }^{2}$, 2002; ALI et al., 2004; CIA et al., 2006).

Com relação à cor da epiderme, os frutos de todos os tratamentos em AM apresentaram menor ICV e maior $h^{o}$, caracterizando assim menor desenvolvimento de cor vermelha que aqueles armazenados somente sob AR (Tabela 2). Isso deve estar relacionado à menor biossíntese e ação do etileno no armazenamento em AM (JAYAS \& JEYAMKONDAN, 2002), pois a mudança na cor durante o amadurecimento de ameixas é um processo dependente da ação desse regulador vegetal

Tabela 1 - Firmeza de polpa e atributos de textura em ameixas 'Laetitia' armazenadas sob refrigeração e em atmosfera modificada por 60 dias e após quatro dias em condição ambiente $\left(20 \pm 2{ }^{\circ} \mathrm{C} / 60 \pm 5 \%\right.$ UR $)$.

\begin{tabular}{|c|c|c|c|c|}
\hline Condicões de armazenamento & Firmeza de polpa (N) & $\begin{array}{l}\text { Força para ruptura da } \\
\text { epiderme }(\mathrm{N})\end{array}$ & $\begin{array}{l}\text { Força para penetração da } \\
\operatorname{polpa}(\mathrm{N})\end{array}$ & $\begin{array}{l}\text { Resistência do fruto à } \\
\text { compressão }(\mathrm{N})\end{array}$ \\
\hline $\mathrm{AR}$ & $17,0 c^{*}$ & $7,31 b$ & $1,23 b$ & $45,1 \mathrm{~b}$ \\
\hline AM & $26,4 b$ & $9,70 \mathrm{a}$ & $1,43 b$ & $36,9 b$ \\
\hline $\mathrm{AM}$ com adição de $\mathrm{CO}_{2}$ & $25,9 b$ & $9,34 \mathrm{a}$ & $1,26 b$ & $39,2 b$ \\
\hline AM com perfuração & $34,0 \mathrm{a}$ & $10,6 \mathrm{a}$ & $1,84 \mathrm{a}$ & $58,5 \mathrm{a}$ \\
\hline \multirow[t]{2}{*}{$\mathrm{CV}(\%)$} & 10,8 & 8,9 & 11,2 & 17,6 \\
\hline & 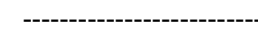 & Após quatro dias & n condição ambiente------- & ---------------------------- \\
\hline AR & $14,5 \mathrm{c}$ & $5,18 \mathrm{c}$ & $1,00 \mathrm{~b}$ & $39,3 b$ \\
\hline AM & $24,9 b$ & $7,88 \mathrm{~b}$ & $1,88 \mathrm{a}$ & $64,6 \mathrm{a}$ \\
\hline $\mathrm{AM}$ com adição de $\mathrm{CO}_{2}$ & $23,3 b$ & $10,4 \mathrm{a}$ & $1,82 \mathrm{a}$ & $59,5 \mathrm{a}$ \\
\hline AM com perfuração & $34,6 a$ & $10,6 a$ & $2,19 \mathrm{a}$ & $70,0 \mathrm{a}$ \\
\hline $\mathrm{CV}(\%)$ & 8,6 & 5,1 & 11,2 & 14,2 \\
\hline
\end{tabular}

* Médias não seguidas pela mesma letra na vertical diferem entre si pelo teste de Tukey, a 5\% de probabilidade de erro. 
Tabela 2 - Cor da epiderme, acidez titulável e sólidos solúveis em ameixas 'Laetitia' armazenadas sob refrigeração e em atmosfera modificada por 60 dias e após quatro dias em condição ambiente $\left(20 \pm 2{ }^{\circ} \mathrm{C} / 60 \pm 5 \% \mathrm{UR}\right)$.

\begin{tabular}{|c|c|c|c|c|}
\hline \multirow[b]{2}{*}{ Condições de armazenamento } & \multicolumn{2}{|c|}{--------Cor da epiderme------------ } & \multirow{2}{*}{$\begin{array}{c}\text { Acidez titulável } \\
\text { (meq de ácido cítrico } 100 \mathrm{~mL}^{-1} \text { ) }\end{array}$} & \multirow{2}{*}{ Sólidos solúveis ( ${ }^{\circ}$ Brix) } \\
\hline & $h^{o}$ & $\operatorname{ICV}(1-4)^{* *}$ & & \\
\hline AR & $52,6 b^{*}$ & $3,51 \mathrm{a}$ & $12,8 b$ & $8,85 \mathrm{a}$ \\
\hline $\mathrm{AM}$ & $59,5 \mathrm{a}$ & $2,86 b$ & $14,8 \mathrm{a}$ & $8,37 \mathrm{a}$ \\
\hline $\mathrm{AM}$ com adição de $\mathrm{CO}_{2}$ & $61,8 \mathrm{a}$ & $3,00 \mathrm{~b}$ & $15,4 \mathrm{a}$ & $8,50 \mathrm{a}$ \\
\hline AM com perfuração & $63,6 \mathrm{a}$ & $2,96 b$ & $15,8 \mathrm{a}$ & $8,60 \mathrm{a}$ \\
\hline \multirow[t]{2}{*}{$\mathrm{CV}(\%)$} & 5,8 & 7,2 & 5,7 & 5,1 \\
\hline & 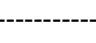 & -----Após & atro dias em condição ambiente-- & ---------------------------------. \\
\hline AR & $30,3 \mathrm{c}$ & $3,98 \mathrm{a}$ & $13,6 \mathrm{a}$ & $9,00 \mathrm{a}$ \\
\hline $\mathrm{AM}$ & $58,7 \mathrm{a}$ & $2,29 \mathrm{c}$ & $14,7 \mathrm{a}$ & $9,10 \mathrm{a}$ \\
\hline $\mathrm{AM}$ com adição de $\mathrm{CO}_{2}$ & $60,5 \mathrm{a}$ & $2,72 \mathrm{c}$ & $14,3 \mathrm{a}$ & $8,25 \mathrm{a}$ \\
\hline AM com perfuração & $48,2 b$ & $3,39 b$ & $15,8 \mathrm{a}$ & $8,45 \mathrm{a}$ \\
\hline $\mathrm{CV}(\%)$ & 11,3 & 6,7 & 17,8 & 4,2 \\
\hline
\end{tabular}

*Médias não seguidas pela mesma letra na vertical diferem entre si pelo teste de Tukey, a 5\% de probabilidade de erro.

$* *$ Valores $1,2,3$ e 4 para $0-25 \%, 26-50 \%, 51-75 \%$ e $>75 \%$ da superfície recoberta com cor vermelha, respectivamente.

(ARGENTA et al., 2003). Os tratamentos em AM, por sua vez, apresentaram diferenças entre si somente aos quatro dias de exposição dos frutos em condição ambiente, quando os frutos armazenados em AM e em AM com adição de $\mathrm{CO}_{2}$ apresentaram menores valores de ICV e $h^{\circ}$ (Tabela 2). Nessas condições de AM houve uma modificação da atmosfera mais intensa, proporcionando pressões parciais de $\mathrm{O}_{2}$ mais baixas e de $\mathrm{CO}_{2}$ mais elevadas, o que possivelmente exerceu um maior controle na biossíntese e ação do etileno.

Os frutos dos tratamentos em AM apresentaram maior acidez do que aqueles armazenados em AR, na saída da câmara (Tabela 2). Os ácidos orgânicos podem ser menos utilizados no metabolismo respiratório durante o armazenamento em $\mathrm{AM}$, devido ao baixo $\mathrm{O}_{2}$ e alto $\mathrm{CO}_{2}$ (CIA et al., 2006).

Não houve diferença entre os tratamentos quando se analisou SS (Tabela 2). Em morangos 'Oso Grande', também não foi observado efeito da AM sobre a manutenção dos SS (BRACKMANN et al., 2005). Possivelmente, a ausência do efeito da AM sobre os SS deve-se ao fato de os açúcares serem metabolizados pela respiração somente após uma acentuada degradação de ácidos orgânicos (SAQUET et al., 1997).

$\mathrm{Na}$ saída da câmara, a ocorrência de podridões apresentou maiores valores no tratamento $\mathrm{AM}$, o qual não diferiu do tratamento $\mathrm{AR}$, e os frutos armazenados em AM com perfurações e AM com adição de $\mathrm{CO}_{2}$ apresentaram menor ocorrência de podridões (Tabela 3). Vários trabalhos reportam o efeito da AM na redução de podridões (JAYAS \& JEYAMKONDAN,
2002; BRACKMANN et al., 2005; CIA et al., 2006), sendo os efeitos do baixo $\mathrm{O}_{2}$ e do alto $\mathrm{CO}_{2}$ sobre a redução na ocorrência de podridões são atribuídos ao aumento na resistência física dos tecidos do fruto, evidenciado principalmente pela maior firmeza de polpa, devido ao efeito dos gases no retardo do amadurecimento (SAQUET et al., 1997; JAYAS \& JEYAMKONDAN, 2002). Contudo, as condições de AM que proporcionaram os menores níveis de $\mathrm{O}_{2} \mathrm{e}$ maiores de $\mathrm{CO}_{2}$ e que, em princípio, deveriam ter menor ocorrência de podridões foram as que apresentaram maior incidência de podridões (Tabela 3). Nesses tratamentos, a combinação do baixo $\mathrm{O}_{2}$ com o alto $\mathrm{CO}_{2}$ deve ter causado um intenso estresse aos tecidos, que pode ser verificado pela alta ocorrência de degenerescência da polpa (Tabela 3 ). Em pressões parciais excessivamente baixas de $\mathrm{O}_{2}$ ou altas de $\mathrm{CO}_{2}$, a integridade do tecido é afetada, e os frutos tornam-se mais suscetíveis ao ataque de patógenos (JAYAS \& JEYAMKONDAN, 2002).

Todos os tratamentos apresentaram alta incidência de degenerescência da polpa (Tabela 3). Contudo, os tratamentos em AM apresentaram os maiores valores de incidência desse distúrbio na saída da câmara. Após quatro dias em condição ambiente, os frutos em AM com perfurações apresentaram menor incidência desse distúrbio quando comparados às demais condições de AM, provavelmente por possuir uma maior pressão parcial de $\mathrm{O}_{2}$ e menor pressão parcial de $\mathrm{CO}_{2}$. A ameixa 'Laetitia' desenvolve degenerescência de polpa durante o armazenamento, principalmente

Ciência Rural, v.39, n.9, dez, 2009. 
Tabela 3 - Incidência de podridões e distúrbios fisiológicos e taxa respiratória em ameixas 'Laetitia' armazenadas sob refrigeração e em atmosfera modificada por 60 dias e após quatro dias em condição ambiente $\left(20 \pm 2{ }^{\circ} \mathrm{C} / 60 \pm 5 \% \mathrm{UR}\right)$.

\begin{tabular}{|c|c|c|c|c|}
\hline Condições de armazenamento & $\begin{array}{l}\text { Podridões } \\
\quad(\%)\end{array}$ & $\begin{array}{c}\text { Degenerescência da polpa } \\
(\%)\end{array}$ & $\begin{array}{c}\text { Rachaduras } \\
\text { (\%) }\end{array}$ & $\begin{array}{l}\text { Taxa Respiratória } \\
\left(\eta \mathrm{mol} \mathrm{CO}_{2} \mathrm{~kg}^{-1} \mathrm{~s}^{-1}\right)\end{array}$ \\
\hline $\mathrm{AR}$ & $23,3 \mathrm{ab} *$ & $71,4 \mathrm{~b}$ & $20,00 \mathrm{a}$ & $187,8 \mathrm{~b}$ \\
\hline AM & $36,7 \mathrm{a}$ & $100 \mathrm{a}$ & $0,00 \mathrm{~b}$ & $535,5 \mathrm{a}$ \\
\hline $\mathrm{AM}$ com adição de $\mathrm{CO}_{2}$ & $20,0 \mathrm{~b}$ & $100 \mathrm{a}$ & $0,00 \mathrm{~b}$ & $556,1 \mathrm{a}$ \\
\hline AM com perfuração & $5,00 \mathrm{c}$ & $100 \mathrm{a}$ & $0,00 \mathrm{~b}$ & $488,7 \mathrm{a}$ \\
\hline $\mathrm{CV}(\%)$ & 17,4 & 5,3 & 9,9 & 12,0 \\
\hline $\mathrm{AR}$ & $1,78 b$ & $\begin{array}{l}74,0 \mathrm{~b} \\
\text {---Após quatro } \mathrm{d}\end{array}$ & $\begin{array}{l}\text { condição aml } \\
0,0\end{array}$ & 397,4a \\
\hline AM & $54,4 \mathrm{a}$ & $91,7 \mathrm{a}$ & 0,0 & $494,2 \mathrm{a}$ \\
\hline $\mathrm{AM}$ com adição de $\mathrm{CO}_{2}$ & $38,0 \mathrm{a}$ & $96,2 \mathrm{a}$ & 0,0 & $438,6 a$ \\
\hline AM com perfuração & $7,85 b$ & $74,3 \mathrm{~b}$ & 0,0 & $421,7 \mathrm{a}$ \\
\hline $\mathrm{CV}(\%)$ & 33,4 & 15,7 & 0,0 & 12,2 \\
\hline
\end{tabular}

*Médias não seguidas pela mesma letra na vertical diferem entre si pelo teste de Tukey, a 5\% de probabilidade de erro.

quando a duração é superior a 30 dias (ARGENTA et al., 2003). Em trabalho recente, observou-se que a ocorrência de degenerescência da polpa, além de aumentar com o avanço do tempo de armazenamento, apresenta um incremento à medida que ocorre um aumento na pressão parcial de $\mathrm{CO}_{2}$, combinado com baixa pressão parcial de $\mathrm{O}_{2}$ (1 a 2kPa) (ALVES, 2009). Em pêssegos e kiwi, o aumento na pressão parcial de $\mathrm{CO}_{2}$ causou incremento na ocorrência de degenerescência da polpa, a qual apresentou correlação positiva com a respiração anaeróbica dos frutos (STEFFENS et al., 2006 e 2007b). Condições de AM em que ocorre grande redução na pressão parcial de $\mathrm{O}_{2}$ e intenso aumento na pressão parcial de $\mathrm{CO}_{2}$ podem causar danos à integridade do tecido (JAYAS \& JEYAMKONDAN, 2002), causando a degenerescência da polpa (STEFFENS et al., 2006). O $\mathrm{CO}_{2}$ atua reduzindo a velocidade do ciclo dos ácidos tricarboxílicos e, em níveis excessivamente elevados, essa redução pode causar acúmulo de ácido succínico devido à inibição da enzima succinato desidrogenase, com consequente desenvolvimento de distúrbios fisiológicos (WATKINS \& ZHANG, 1998). O desenvolvimento de escurecimento da polpa ocorre em condições de armazenamento que podem reduzir o metabolismo energético e o conteúdo de fosfolipídios, com consequente descompartimentalização intracelular (SAQUET et al., 2003; VELTMAN et al., 2003).

A incidência de rachaduras somente foi observada nos frutos armazenados em AR e na avaliação realizada na saída da câmara (Tabela 3). Em maçãs 'Royal Gala' e 'Galaxy', o baixo $\mathrm{O}_{2}$ e o alto $\mathrm{CO}_{2}$ reduziram a ocorrência de rachaduras, em relação ao armazenamento em AR (BRACKMANN et al., 2008), corroborando os resultados do presente trabalho. Segundo esses autores, a ocorrência desse distúrbio está fortemente relacionada ao retardo no amadurecimento dos frutos.

Na saída da câmara, a taxa respiratória foi maior nos frutos armazenados em $\mathrm{AM}$, não havendo diferença entre tratamentos após quatro dias de exposição em condição ambiente (Tabela 3 ). Em maçãs 'Royal Gala', o alto $\mathrm{CO}_{2}$ também aumentou a taxa respiratória (BRACKMANN et al., 2000), corroborando os resultados obtidos no presente trabalho. A taxa respiratória aeróbica pode ser reduzida pelo baixo $\mathrm{O}_{2} \mathrm{e}$ pelo alto $\mathrm{CO}_{2}$, mas, em condições extremas de baixo $\mathrm{O}_{2}$ e/ou alto $\mathrm{CO}_{2}$, pode aumentar a respiração anaeróbica, resultando assim em dano às células vegetais (WATKINS \& ZHANG, 1998).

\section{CONCLUSÃO}

A atmosfera modificada (AM) com acúmulo de $\mathrm{CO}_{2}$, apesar de retardar o amadurecimento dos frutos, não é adequada para o armazenamento de ameixas 'Laetitia', pois aumenta a incidência de degenerescência da polpa. A AM com perfurações no filme apresenta potencial de utilização no armazenamento de ameixas 'Laetitia'. Contudo, novos estudos devem ser realizados com o objetivo de avaliar tecnologias complementares à AM.

\section{AGRADECIMENTOS}

Os autores agradecem ao Conselho Nacional de Desenvolvimento Científico e Tecnológico (CNPq) e à Fundação de Amparo à Pesquisa e Desenvolvimento Científico e Tecnológico de Santa Catarina (FAPESC), pelo apoio financeiro.

Ciência Rural, v.39, n.9, dez, 2009. 


\section{REFERÊNCIAS}

AKBUDAK, B.; ERIS, A. Physical and chemical changes in peaches and nectarines during the modified atmosphere storage. Food Control, Amsterdam, v.15, p.307-313, 2004. Disponível em: <http:// www.sciencedirect.com/science? ob=ArticleURL\& udi=B6T6S$48 \mathrm{TMGBC} 1 \&$ user $=686176 \&$ rdoc $=1 \&$ fmt $=\&$ orig $=$ search \& sort $=\mathrm{d} \&$ 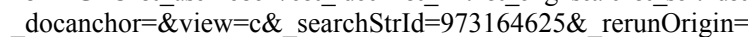 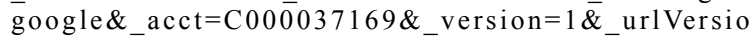 $\mathrm{n}=0$ \&_userid $=686176 \& \mathrm{md} 5=\mathrm{ac} 186 \mathrm{~d} 502 \mathrm{ea} 943 \mathrm{af} 393441 \mathrm{dfb} 707 \mathrm{adff}>$. Acesso em 04 de ago de 2009. doi:10.1016/S0956-7135(03)00082-3.

ALI, Z.M. et al. Low temperature storage and modified atmosphere packaging of carambola fruit and their effects on ripening related texture changes, wall modification and chilling injury symptoms. Postharvest Biology and Technology, Amsterdam, v.33, n.2, p.181-192, 2004. Disponível em: $<\mathrm{ht} \mathrm{t}$ : / / w w w s c i e n c e d i e c t. c o m / science? ob=ArticleURL\& udi=B6TBJ-4CJCWJM$5 \&$ user $=686176 \&$ rdoc $=1 \&$ fmt $=\&$ \& orig-search\&_sort $=\mathrm{d} \&$ docanchor $=\&$ vie 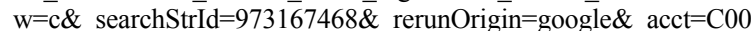 $0037169 \&$ \& version $=1 \&$ \&_ur 1 Version $=0 \&$ userid $=6$ $86176 \& \mathrm{md} 5=3553 \mathrm{a} 85 \mathrm{~d} 6 \mathrm{c} 48 \mathrm{a} 07 \mathrm{~d} 550 \mathrm{cb} 68 \mathrm{e} 6 \mathrm{a} 64 \mathrm{f} 3 \overline{\mathrm{da}}>$. Acesso em 04 de ago de 2009. doi:10.1016/j.postharvbio.2004.02.007.

ALVES, E.O. Manejo da temperatura e do etileno na conservação de ameixas 'Laetitia' em armazenamento refrigerado e atmosfera controlada. 2009. 61f. Dissertação (Mestrado em Produção Vegetal) - Universidade do Estado de Santa Catarina, SC.

ARGENTA, L.C. et al. Ripening and quality of 'Laetitia' plums following harvest and cold storage as affected by inhibition of ethylene action. Pesquisa Agropecuária Brasileira, Brasília, v.38, n.10, p.1139-1148, 2003. Disponível em: <http:// w w w. s c i e 1 o.b r / s c i e lo.ph p ? p i d = S $0100-$ $204 X 2003001000002 \&$ script $=$ sci arttext $>$. Acesso em 04 de ago de 2009. doi: 10.1590/S0100-204X2003001000002.

BRACKMANN, A. et al. Armazenamento de maçã cv. Royal Gala em atmosfera controlada com alta pressão parcial de $\mathrm{CO}_{2}$. Revista Brasileira de Armazenamento, Viçosa, v.25, n.2, p.3-8, 2000 .

BRACKMANN, A. et al. Armazenamento de ameixas 'Reubennel' e 'Pluma 7' em frigoconservação intermitente e atmosfera controlada. Revista Científica Rural, Bagé, v.6, n.1, p.71-76, 2001 .

BRACKMANN, A. et al. Armazenamento de morangos 'Oso Grande' em atmosfera modificada enriquecida com $\mathrm{CO}_{2}$. Revista Científica Rural, Bagé, v.10, n.1, p.16-22, 2005.

BRACKMANN, A. et al. Manutenção da qualidade pós-colheita de maçãs 'Royal Gala' e 'Galaxy' sob armazenamento em atmosfera controlada. Ciência Rural, Santa Maria, v.38, n.9, p.2478-2484, 2008.

CIA, P. et al. Modified atmosphere packaging for extending the storage life of 'Fuyu' persimmon. Postharvest Biology and Technology, Amsterdam, v.42, p.228-234, 2006. Disponível em: <http://www.sciencedirect.com/ science? ob=ArticleURL\& udi=B 6TBJ-4KWK10R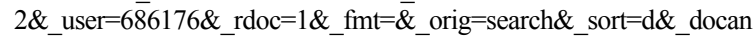 chor $=\&$ view $=c \&$ csearchStrId $=973169876 \&$ \& rerunOrigin $=$ go ogle\&_a c c t $=\overline{\mathrm{C}} 000037169 \&$ \& version $=1 \&$ \&_ur $1 \mathrm{~V}$ ersion $=0 \& \_$userid $=686176 \& \mathrm{md} 5=\mathrm{e} 7 \mathrm{c} 44 \mathrm{eaea} 793 \mathrm{a} 17 \mathrm{c} 9 \mathrm{cf0a} 4 \mathrm{cad} 88 \mathrm{c} 64 \mathrm{cb}>$. Acesso em 04 de ago de 2009. doi: 10.1016/ j.postharvbio.2006.06.016.
JAYAS, D.S.; JEYAMKONDAN, S. Modified atmosphere storage of grains, meats, fruits and vegetables. Biosystems Engineering, Amsterdam, v.82, n.3, p.235-251, 2002. Disponível em: $<$ http:// www.sciencedirect.com/science? ob=ArticleURL\& udi=B6WXV46 HDMGH-1\&_user $=686176 \&$ _rdoc $=1 \&$ fmt $=\&$ _orig $=$ search $\&$ sort $=$ d\& docanchor $=\& v i e w=c \&$ searchStrId $=97317$ $4856 \&$ rerunOrigin $=$ google $\&$ _acct $=$ C 000037169 $\& \quad$ vers i o $=1 \&$ u r 1 Version $=0 \&$ u s e ri $\mathrm{d}=686176 \& \mathrm{md} 5=3 \mathrm{dbe} 9 \mathrm{c} 50 \mathrm{aadf0f} 209 \mathrm{dcdae} 3 \mathrm{e} 9 \mathrm{ccc} 16 \mathrm{e} 0>$. Acesso em 04 de ago de 2009. doi:10.1006/bioe.2002.0080.

JERONIMO, M.E. et al. Conservação pós-colheita de mangas 'Tommy Atkins' armazenadas sob atmosfera modificada. Semina: Ciências Agrárias, Londrina, v.28, n.3, p.417426, 2007.

SAQUET, A.A. et al. Armazenamento de maçã 'Gala' sob diferentes temperaturas e concentrações de oxigênio e gás carbônico. Ciência Rural, Santa Maria, v.27, n.3, p.399405, 1997.

SAQUET, A.A. et al. Energy metabolism and membrane lipid alterations in relation to Brown heart development in 'Conference' pears during delayed controlled atmosphere storage. Postharvest Biology and Technology, Amsterdam, v.30, p.123-132, 2003.

STEFFENS, C.A. et al. Escurecimento da polpa e respiração de pêssegos em função das condições de armazenamento. Revista Brasileira de Agrociência, Pelotas, v.12, n.1, p.71-75, 2006.

STEFFENS, C.A. et al. Taxa respiratória de frutas de clima temperado. Pesquisa Agropecuária Brasileira, Brasília, v.42, n.3, p.313-321, 2007a. Disponível em: <http:// www.scielo.br/pdf/pab/v42n3/03.pdf $>$. Acesso em 04 de ago de 2009. doi: 10.1590/S0100-204X2007000300003.

STEFFENS, C.A. et al. Degenerescência da polpa e respiração de quivi cv. Bruno em função das condições de armazenamento. Ciência Rural, Santa Maria, v.37, n.6, p.1621-1626, 2007b. Disponível em: <http://www.scielo.br/scielo.php?pid=S0103$84782007000600018 \&$ script $=$ sci arttext $>$. Acesso em $04 \mathrm{de}$ ago de 2009. doi: 10.1590/S0103-84782007000600018.

STEFFENS, C.A. et al. Respiração, produção de etileno e qualidade de maçãs 'Gala' submetidas ao dano mecânico e tratadas com 1-metilciclopropeno. Ciência Rural, Santa Maria, v.38, n.7, p.1864-1870, 2008. Disponível em: <http://www.scielo.br/ scielo.ph ? s cript $=$ sci_arttext \& pid=S 0103 $84782008000700010>$. Acesso em 04 de ago de 2009. doi: 10.1590/S0103-84782008000700010.

STEFFENS, C.A. et al. Aplicação pré-colheita de reguladores vegetais visando retardar a maturação de ameixas 'Laetitia'. Ciência Rural, Santa Maria, 39,n.5, p.1369-1373, 2009. Disponível em: <http:// www.scielo.br/scielo.php? script $=$ sci arttext\&pid $=$ S0103$84782009000500010 \& \operatorname{lng}=\mathrm{pt} \& \mathrm{nrm}=\mathrm{iso} \& \mathrm{t} \operatorname{lng}=\mathrm{pt}>$. Acesso em 04 de ago de 2009. doi:10.1590/S0103-84782009005000101.

VELTMAN, R.H. et al. Internal browning in pear fruit (Pyrus communis L. cv Conference) may be a result of a limited availability of energy and antioxidants. Postharvest Biology and Technology, Amsterdam, v.28, p.295-302, 2003.

WATKINS, C.B.; ZHANG, J. Metabolic responses fruit to carbon dioxide. Acta Horticulturae, Leuven, n.464, p.345-350, 1998 .

Ciência Rural, v.39, n.9, dez, 2009. 